\title{
Die byzantinische Medizin als Konzept einer spätantiken christlichen Heilkunde
}

\begin{abstract}
Byzantine Medicine as a Concept of Late Ancient Christian Healing Art. The great success of Greco-Roman medicine - in its main stream a brilliant combination of humoral pathology and dietetics canonized by Galen of Pergamon in the 2nd century CE - is probably the most surprising phenomenon of conceptual longevity in the history of Western culture and civilization. Its decline begins as late as in the early 17th century, when William Harvey describes the circulation of blood on the basis of the new experimental method, initiating not only the collapse of Galen's theory of blood circulation, but also of humoral physiology and pathology in general. Only then, i.e., more than 1500 years after Galen and 2000 years after Hippocrates, new theoretical concepts of medicine appear on the horizon, gradually replacing medical thinking of antiquity. However, the evolution of Greco-Roman medicine was not a straightforward process; it was strongly influenced by changes in language and dramatic institutional and political changes after the separation of the Roman Empire at the end of the 4th century. Byzantine medicine in the East encompasses the common medical practices of the empire from about 400 to $1453 \mathrm{AD}$, compiling and standardizing medical knowledge and wisdom (iatrosophia) into new Greek textbooks.
\end{abstract}

\section{Einleitung}

Die Wirkmächtigkeit der antiken Medizin, so wie sie als Qualitäten- und Humoralkonzept und als Kunst der menschlichen Lebensführung (diaita) durch Galen von Pergamon im 2. Jahrhundert n. Chr. auf dem Boden einer damals bereits 400jährigen Tradition kanonisiert wurde, ist vielleicht eines der erstaunlichsten Phänomene der westlichen Kultur und Wissenschaftsgeschichte überhaupt. Erst mit William Harveys experimentell begründeter Beschreibung des großen Blutkreislaufs und der Durchsetzung der experimentellen Methode beginnt der Niedergang der bis dahin zwar mit Veränderungen, Korrekturen und Erweiterungen versehenen, ansonsten aber in ihrer konzeptionellen Grundlegung konstant gebliebenen Medizin der Antike. Nun erst, in der zweiten Hälfte des 17. Jahrhunderts, 1500 Jahre nach Galen und fast 2000 Jahre nach Hippokrates, treten neue Kon-

\section{Wolfgang Uwe Eckart, Ruprecht-Karls-Universität Heidelberg}

๑ OpenAccess. ( 2021 Wolfgang Uwe Eckart, publiziert von De Gruyter. (cc) BY-NC-SA Dieses Werk ist lizenziert unter einer Creative Commons Namensnennung - Nicht kommerziell - Weitergabe unter gleichen Bedingungen 4.0 International Lizenz. https://doi.org/10.1515/9783110713336-010 
zepte an ihre Stelle. Die Transition der antiken Medizin verlief nicht geradlinig. Sie unterlag sowohl sprachlich als auch institutionell und politisch den dramatischen Veränderungen, denen das römische Kaiserreich und seine Nachfolgegebilde seit Galens Zeiten unterworfen war. Es handelt sich hierbei um die Teilung des Mittel-, Süd- und Westeuropa, Nordafrika und Vorderasien umfassenden römischen Kaiserreichs in ein westliches und östliches Imperium im 4. Jahrhundert unserer Zeitrechnung, um die kulturelle und politische Entwicklung des byzantinischen Großreichs zwischen dem 4. Jahrhundert und seinen Untergang in der Mitte des 15. Jahrhunderts. Im vorliegenden Beitrag soll der Blick auf Byzanz fallen, in dem Ärzte und Philosophen das sprachliche Erbe der griechisch verfassten medizinischen Überlieferung der Antike antraten, zum Teil aber auch inhaltlich bereicherten und weiterentwickelten. Besonders herausgehoben werden soll dabei der christliche Charakter der byzantinischen Medizin, wie er der christlichen Staatsverfassung entsprach und sich besonders im Hospitalwesen und in der Schutzheiligenverehrung manifestierte.

\section{Medizinische Polypragmasie: Christus, Heilige und Konzepte}

Byzanz, das faszinierende fast tausendjährige Großreich des Mittelmeerraums und Vorderasiens zwischen Spätantike und Mittelalter, ist für die europäische Medizingeschichte bis in die frühe Neuzeit von überragender Bedeutung (Congourdeau 2004; Grumel 1949; Miller 1997; Temkin 1962). Seine Ärzte stehen ganz in der Tradition ihrer großen antiken Vorbilder: Hippokrates, Galen, Dioskurides, Theophrast und vieler mehr. Es ist ihr Erbe, das sie bewahren. Es ist umfassend und geschlossen im medizintheoretischen, humoral- und qualitätenpathologisch, diätetisch bestimmten Denkhorizont seiner Zeit. Und auch in praktischer Hinsicht genügt es ihnen in der Pulslehre, in den Entleerungstechniken, in der Chiropraxis und Chirurgie, in der Inspektion der Kranken, der medikamentösen Therapeutik und der Prognose ihrer Leiden.

Auch die Medizin ist durch und durch geprägt vom christlichen Charakter der byzantinischen Gesellschaft. Christos Philantropos Pantokrator, der menschenfreundliche Allherrscher Christus, der heilende Heiland, bestimmt das Denken der Patienten und der christlichen Ärzte jener Epoche. Diese Auffassung war christologisch überaus modern, denn die Übertragung des Titels Pantokrator (Allherrscher) auf Gottes Sohn hatte sich, obwohl in der christlichen Überlieferung des Neuen Testaments bereits vorbereitet, ausdrücklich erst im Verlauf des 4. Jahrhunderts vor dem Hintergrund christologischer Kontroversen (Arianismus- 
Streit) vollzogen; durch die Verehrung Jesu Christi als Christus medicus im Westen oder eben als Pantokrator soter im Osten sollte dessen vollkommene göttliche Natur betont werden, wie es vor allem Athanasius von Alexandrien lehrte (Knipp 1998, S. 1-4). Im späten römischen Kaiserreich kam es in diesem Zusammenhang zunächst noch nicht zu maßgeblichen Versuchen, Wesensgleichheiten zwischen Christus medicus und dem paganen Heilgott Asklepios zu suchen oder herzustellen. Wo dies doch geschah, so etwa bei Origenes oder Arnobius, wurden solche Vorstellungen bekämpft (Tertullian, Eusebius) (Edelstein \& Edelstein 1945, S. 132-133). Asklepios soter blieb der heidnische körperheilende Gott ohne erlösende Wirkung im transzendenten Sinn, während Christus medicus zwar als medicus humilis, der menschgewordene niedere Arzt, aber eben doch auch als Sohn Gottes nicht nur die Kraft der körperlichen Heilung, sondern auch die der seelischen Erlösung ( $\sigma \omega \tau \eta \rho i ́ \alpha)$ innewohnte (Abb. 1). Gleichwohl setzt bereits im 3. Jahrhundert das allmähliche Verblassen der Figur des Asklepios zugunsten derjenigen eines heilenden Christus ein. Besonders unter dem Einfluss des Augustinus entfaltet sich dann die Figur des Heiland Christus (Arbesmann 1954; Constantelos 1976; Gollwitzer-Voll 2007; Markschies 2005, 2016).

Hinzu traten in der christlichen Glaubenswelt die Heiligen (Damian of Sinai [Erzbischof] 2007), allen voran die Gottesmutter Maria, aber auch die in Byzanz verehrten philanthropisch-heilkundigen „Anargyroi“ ('Ayı Avópyvpos, Agioi Anárgyroi, die Heiligen, die kein Geld nehmen) Cosmas und Damian (beide gest. um 287 n. Chr.), Lukas der Evangelist, Arzt und Maler (1. Jahrhundert), Zenaida von Tarsus und Philonella (beide um 100 n.Chr.), der Tierheiler Tryphon auf Campsada (3. Jh. n. Chr.), Cyros und Johannes im frühen 4. Jahrhundert, bis heute in Apulien hochverehrt, Diamedes von Tarsus (um 300 n.Chr.), Pantaleon aus Nikomedia (um 300 n. Chr.) oder Sampson der Fremdenfreundliche (gest. um 530 n.Chr.). $\mathrm{Zu}$ erwähnen sind exemplarisch aber auch die bestimmten Orten zugeschriebenen Schutzheiligen Achatius von Byzanz (gest. ca. 303/4) gegen Kopfweh und Todesangst, Alexius von Edessa (gest. ca. 417 in Rom) gegen die Pest, epidemische Krankheiten und Naturkatastrophen, Cyprian von Antiochia (gest. 304, in Nikomedia/Izmir) gegen Dämonen und Verhexung oder der Hyperhagios und Thaumaturg Nikolaus von Myra (ca. 283 - ca. 348 in Myra/Demre), der noch heute besonders in der Orthodoxie als Schutzheiliger vieler Berufe und Gewerke, im Westen vor allem als vorweihnachtlicher Gabenbringer verehrt wird. Noch heute werden die Heiligen Anargyroi an vielen Orten im Einflussbereich der orthodoxen und koptischen Kirche, aber auch im Westen verehrt. In Athen ist ein Stadtteil nach ihnen benannt ('Ayı ı Avópyupoı). Die christlichen Krankenhäuser des byzantinischen Reichs waren oft Klöstern angeschlossen, und christliche Philanthropie motivierte ihre Stifter und Förderer, nach denen sie häufig benannt wurden. Gerade im Stiftungswesen der Krankenhäuser und Klöster gingen Schutzheili- 


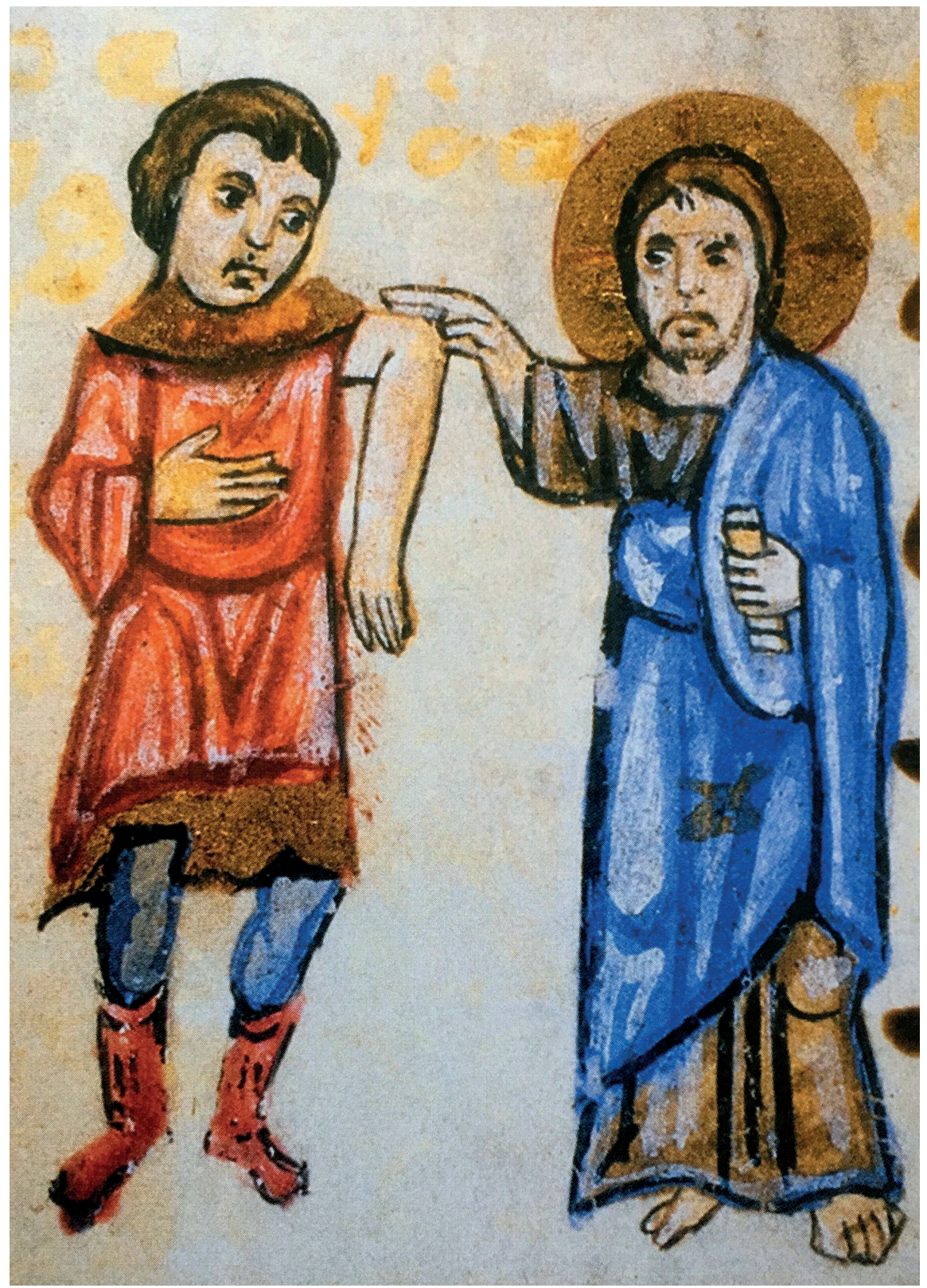

Abb. 1: „Heilung des Mannes mit der verdorrten Hand“ - Nach Mt. 12,10. Homilien des Johannes Chrysostomos zum Matthäus-Evangelium. Pergamenthandschrift, mittelbyzantinisch, um 1150, fol. 347r. (München, Bayer. Staatsbibl., Cod. Graec I) (Bildsammlung IGEM, Heidelberg) 
genverehrung und politische Heiligenverehrung (Schreiner 2006) eine enge Verbindung ein, denn Kaiser und Kaiserinnen konnten auch ohne Martyrium bereits zu Lebzeiten zu Staatsheiligen erhoben werden. Neben den christlichen Ärzten wirkten indessen auch jüdische und vom 7. Jahrhundert an muslimische Ärzte, die gleichermaßen ihrem Glauben verbunden und entsprechend tätig waren. Den medizinischen Inhalten, nach denen prognostiziert und therapiert wurde, tat dies keinen Abbruch, insofern sie nicht paganen Heilvorstellungen der antiken Welt verbunden oder verpflichtet waren.

Rational-konzeptionell war die byzantinische Medizin der antiken Humorallehre verpflichtet. In der bereits in der Naturlehre einiger Vorsokratiker angelegten, aber zuerst durch Hippokrates von Kos (ca. 460-370) ausformulierten und durch Galen von Pergamon (129-ca. 200) kanonisierten antiken Säftelehre, die das medizinische Denken und ärztliche Handeln in der byzantinischen Medizin und weit über sie hinaus - bestimmt, ist Gesundheit durch ein ausgeglichenes

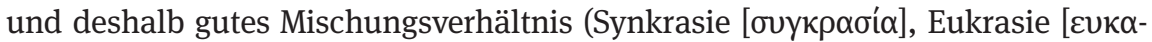
$\rho i \alpha$, temperies) der vier Kardinalsäfte des Menschen (die res naturales Blut, gelbe Galle, schwarze Galle, Schleim) und der ihnen zugewiesenen Qualitäten (warm/feucht, warm/trocken, kalt/trocken, kalt/feucht) bestimmt. Der menschliche Körper ist nach Galen gesund, wenn er in all seinen Teilen ausgeglichen, temperatum ist. Krank ist er, wenn seine Säfte- und Qualitätenmischung unausgeglichen ist (Dyskrasie [ $\Delta v \sigma \kappa \rho \alpha \sigma i ́ \alpha]$, intemperies). Ziel ärztlicher Kunst ist es, das ausgeglichene, gesunde Mischungsverhältnis der Säfte und Qualitäten wieder herzustellen, sei es durch entleerende Maßnahmen (Aderlass, Brechen, Abführen) oder durch die medikamentöse Therapie, die vom Prinzip des contraria contrariis (mit Gegensätzlichem Behandeln: z. B. kalt gegen warm) geleitet wird. Die sechs res non naturales (Luft, Nahrung, Schlaf, Bewegung, Stoffwechsel, Gemütsregungen) kann der Mensch im Rahmen der allgemeinen Diätetik selbst beeinflussen, um seine Gesundheit zu bewahren.

Bei der Diätetik handelte es sich um die Lehre von der gesunden Lebensführung, wie sie dem Patienten selbst anbefohlen war. Die hippokratische Medizin verstand die Bewahrung der Gesundheit als eine ihrer zentralen Aufgaben: Diätetik stellte zusammen mit der medikamentösen Therapie und der Chirurgie eine der drei konstitutiven Interventionsformen der Medizin dar. Die antike Philosophie begründete das für die Diätetik zentrale Prinzip vom (Mittel-)Maß - als geordnete seelische und körperliche Lebensführung - ethisch und ästhetisch. Die individuell angepasste Lebensführung, die unter Berücksichtigung der vier Jahreszeiten und der vier Lebensalter bestimmt wurde, sollte dazu beitragen,

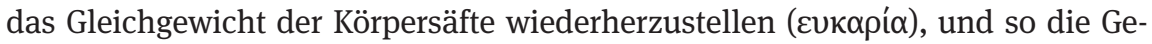
sundheit gewährleisten. Die der antiken Diätetik eigenen Bereiche der sex res non naturales galten ungebrochen auch in Byzanz weiter. 
Auch wenn die Ablehnung der Magie, ihrer Heilmittel und Praktiken in den Händen von Magiern oder Ärzten in der römischen Kaiserzeit nachgerade zum Etikett des rational handelnden Arztes erhoben wurde (Ulpian, Galen) (Helm 2005), gehörten doch bei einigen Ärzten der späteren Antike und in Byzanz, etwa bei Alexander von Tralleis, solche iatromagischen Methoden (Rothschuh 1978) durchaus zur geläufigen Heiltätigkeit und wurden wohl auch vom Publikum so erwartet (Vikan 1984). Religiöse und magische Heilverfahren ergänzten sich dabei und nutzten magische Objekte, Amulette, Zaubersprüche oder ähnliche Besprechungsformeln ebenso wie exorzistische Verfahren, um krankmachende Dämonen oder Teufel aus dem Körper der Patienten zu vertreiben (László 2010). Daneben war die Vorstellung magischer Kräfte auch in der engeren Ernährungsdiätetik durchaus verbreitet (z.B. Knoblauch gegen den bösen Blick), wobei vermutlich vor allem auf Plinius zurückgegriffen wurde.

Aus dem Konglomerat der medizinischen Konzepte ergeben sich auch Rückschlüsse auf die Vorstellungen von Krankheit und Gesundheit in einer zwar christlich verfassten Gesellschaft, die aber gerade in ihrem medizinischen Wissen noch überaus stark der Antike und ihrer Heilkunst verpflichtet war. Zwar waren die paganen Kulte der theurgischen Medizin mit Asklepios und seinen Heilstätten, den Asklepieien, aufgelöst beziehungsweise durch die Figur des heilenden Christus medicus ersetzt worden und an die Stelle der Asklepieien sehr häufig christliche Basiliken getreten. Man betete dort nun zu Christus dem Heiler und zu den ihm nahestehenden Heiligen mit ihren besonderen Schutzfunktionen und legte nach erfolgter Heilung dort auch Votivgaben ab. In der rationalen Medizin und Gesundheitsvorstellung blieb allerdings zunächst alles beim Alten. Krankheit war eine gestörte Mischung der Säfte, um deren Gleichgewicht der Arzt mit allen ihm zur Verfügung stehenden Mitteln bemüht war. Dabei fiel auch den Patienten weiterhin eine zentrale Rolle im Kontext der Diätetik zu. Sie hatten in allen körperlichen Dingen, die sie selbst beeinflussen konnte, für ein ausgeglichenes Maß zu sorgen. Blieben die Heilmaßnahmen des Arztes erfolglos, konnte im Gebet Heilung und Heil von Christus und den Heiligen erbeten werden. Dies bedeutete zugleich aber auch, dass an die Kraft eines göttlichen Einflusses auf den menschlichen Körper in Gesundheit und Krankheit geglaubt wurde. Neben solche Vorstellungen, die einerseits dem rationalen Erbe der antiken Medizin entsprachen, andererseits dem Christentum verpflichtet waren, traten - wie übrigens auch in der antiken Medizin - magische Heilmittel und Heilrituale und die Vorstellung, dass mit der Applikation solcher Mittel Krankheit aus dem Körper vertrieben werden konnte. So nahm auch das Element der magischen Sympathie und Antipathie in der Medizin noch eine Rolle ein, wenn alle anderen Heilmittel erschöpft waren oder wenn der Patient nach ihnen verlangte. Irrationale Aspekte von Krankheitsvorstellung und Heilung beinhaltete aber auch die vorgestellte 
Konkurrenz von Christus, Maria und der Familie der Heiligen mit Dämonen und Teufeln. Auch Mittel der exorzistischen Austreibung von Krankheits-Dämonen gehörten, wenngleich randständig, noch in den Vorstellungsbereich von „krank sein“ und „gesund werden“.

Das Krankheitsspektrum jener Zeit zwischen dem 4. und 15. Jahrhundert dürfte sich von dem unserer Lebenswelt kaum wesentlich unterschieden haben. Aber natürlich gab es besondere Probleme im Mittelmeerraum bis tief hinein nach Vorderasien und Mesopotamien. Fieberkrankheiten spielten eine erhebliche Rolle, sicher die Malaria, Aussatzkrankheiten wie die Lepra und eine Vielzahl parasitärer Infektionen. Die erste große Pest (,Justinianische Pest“, Ausbruch 541/ 542 n. Chr.), von der wir auch in der mitteleuropäischen Seuchengeschichte wissen, fand zur Regierungszeit des Justinian statt, forderte Millionen Tote und wütete in ihren Ausläufern bis ins 8. Jahrhundert. Die Ärzte waren so hilflos wie Jahrhunderte später - 1348 - anlässlich der Katastrophe des „Schwarzen Todes“. Viele Krankheiten aber konnten behandelt werden, mit Medikamenten, chiropraktisch, chirurgisch oder durch eine Umstimmung der Lebenshaltung, der Diätetik. Die Menschen vertrauten ihren Ärzten so stark oder so wenig, wie wir es heute tun. Das ärztliche Handeln war damals wie heute ein Wagnis, viele Krankheiten waren gefährlich und manche führten zum Tod, wie das Leben ohnehin.

\section{Bewahrung des antiken Erbes}

Für die Medizingeschichte beginnt mit dem politischen und kulturellen Zerfall des Römischen Kaiserreichs im 4. Jahrhundert und dem Aufblühen von Byzanz die Phase der Rezeption des antiken Erbes. Bei allen Problemen, die chronologische Einteilungen kultureller Epochen aufwerfen, scheint doch eine Zweiteilung der byzantinischen Medizin möglich, die sich wiederum an einem bestimmten politischen Ereignis orientiert: der Eroberung Alexandrias durch die Araber im Jahre 642. Die erste Phase der byzantinischen Medizin - von der Reichsteilung Roms 395 nach dem Tod von Theodosius I. (347-395) bis zur Eroberung Alexandrias 641 durch islamische Truppen - kann als spätalexandrinisch bezeichnet werden, befand sich doch ihr kulturelles und geistiges Zentrum in Alexandria. Inhaltlich ist diese Phase als die einer kompilierenden und zusammenfassenden Rezeption der klassisch-antiken Medizinkonzepte zu deuten. Ihre wichtigsten Vertreter waren Oreibasios von Pergamon, Aëtios von Amida, Alexandros von Tralleis und Paulos von Aigina. Die zweite Phase der byzantinischen Medizin (643-1453) reicht vom ausgehenden 7. Jahrhundert bis zum Fall Konstantinopels (1453) und lässt sich als Epoche charakterisieren, die stark klinisch orientiert ist. Nach der 
Phase der Kompilation geht es nun um die weitere Ausdifferenzierung der diagnostischen Methodik insbesondere in der Pulslehre, aber auch in der Uroskopie, um die Erweiterung des konservativ-therapeutischen Handlungsspektrums ( $M a-$ teria medica) sowie um die Ausdifferenzierung und Perfektionierung der chirurgischen Technik. Insbesondere auf dem letzten Gebiet sind in dieser Hochphase der byzantinischen Medizin Fertigkeiten und Methoden entwickelt worden, die bereits vieles von dem vorwegnahmen, was in der frühen Neuzeit erst mühsam wiederentdeckt und -eingeführt werden musste. Hauptvertreter dieser Phase waren im 11. Jahrhundert der Arzt Michael Psellos, dessen Zeitgenosse Simeon Seth sowie am Ausgang des Jahrhunderts der Chirurg Niketas. Im 13. und 14. Jahrhundert sind es vor allem der aus Alexandria gebürtige Nikolaos Myrepsos und Johannes Aktuarios.

\section{Die erste Phase der byzantinischen Medizin}

Der wichtigste Kompilator in der Frühphase der byzantinischen Medizin war zweifelsohne der aus Pergamon gebürtige „Iatrosophist“ Oreibasios (ca. 325 400). Er hat seine Erziehung und ärztlich-arztphilosophische Ausbildung sicherlich auch in Pergamon erhalten - einer Stadt, der auch noch in der späten Antike der Ruf vorausging, dass aus ihr die besten Ärzte kämen. Der Weg führte ihn dann nach Alexandria in die Nähe des Arztes und Philosophen Zenon von Kypros (4. Jh.). Dessen Einfluss verdankte Oreibasios wohl auch eine Anstellung als Leibarzt am Hofe des späteren Kaisers Flavius Claudius Julianus [Julian Apostata] (331-363), den er vermutlich auf seinem Kriegszug nach Gallien und ins oberrheinische Germanien (356-360) begleitete. Um 360 wirkte Oreibasios in Konstantinopel als Quaestor (Magistrat), war in die Ausrufung Julians zum Kaiser (361) involviert und begleitete Julian nach Mesopotamien (363). Oreibasios war seit 355 nicht nur Leibarzt Julians, sondern zugleich für dessen umfangreiche Bibliothek verantwortlich, die er erweiterte. In der Folgezeit entwickelte sich eine Freundschaft zwischen den beiden Männern. Oreibasios wurde ein enger Vertrauter des Kaisers, der nach seiner Thronbesteigung 361 auf eine Restauration der heidnischen Kulte hinarbeitete. Byzantinische Historiker berichten, dass Oreibasios für seinen Kaiser und Freund auch das Orakel der Pythia befragt habe, das ihm allerdings den baldigen Tod Julians weissagte. Ein wechselhaftes Schicksal führte den Arzt unter den Nachfolgern Julians, Flavius Valens und Valentinian I., zunächst in die Verbannung, bald aber wieder zurück in seine Heimat, wo er zu Beginn des 5. Jahrhunderts hochbetagt und angesehen starb. Das Hauptwerk des Oreibasios bildet eine 70 Bücher (nur 25 sind erhalten) umfassende Medizinische

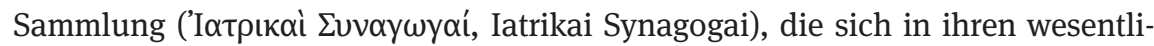


chen Teilen als eine Kompilation der Werke des Galen erweist. Aber auch andere Textstellen der „besten Ärzte“ (Dioskurides, Rufus von Ephesos und viele andere) sind beinhaltet. Einen Extrakt aus diesem umfangreichen Werk stellt die Synopsis pro Eustathion dar, in der die wesentlichen Tatsachen der ärztlichen Kunst lehrbuchartig zusammengefasst werden. Wichtig sind seine Überlieferungen chirurgischer Texte des Antyllos und des Heliodoros (beide im 2. Jahrhundert n.Chr.), denen wir Beschreibungen des Luftröhrenschnittes oder auch der Operation des grauen Stars verdanken. Der breiten Popularisierung schließlich diente ein ebenfalls Oreibasios zuzuschreibendes Rezeptbuch „leicht beschaffbarer Mittel“ (Euporista). Es war dem befreundeten Historiker und Philosophen Eunapios von Sardeis (345-420) gewidmet und für Laien bestimmt. Oreibasios schrieb griechisch, beanspruchte keine Originalität, ist aber als Kompilator älterer Werke von unschätzbarem Wert.

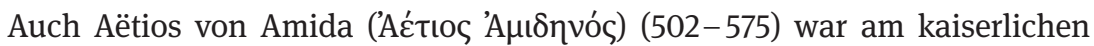
Hof tätig. Aus Amida, dem heutigen Diyabakir, einer kleinen Stadt am Oberlauf des Tigris stammend, erwarb Aëtios seine ärztlichen Kenntnisse an der seit dem 1. Jahrhundert v. Chr. hoch angesehenen Medizinschule von Alexandria. Als junger Arzt ging er nach Konstantinopel und fand dort am Hofe Justinians I. (482565) als Comes obsequii (Beamter in der unmittelbaren Gefolgschaft des Kaisers, vermutlich in seiner Funktion als Arzt) Aufnahme. Aëtios war, anders als Oreibasios, Christ und ist uns als Verfasser einer 16 Bücher umfassenden Kompilation bekannt, die in ihrem überwiegenden Teil auf Galen, aber auch auf Rufus von Ephesos (ca. 80-150 n.Chr.), Pedanius Dioskurides (1. Jh. n.Chr.) und anderen Autoren fußt. Das Werk des Aëtios, das wegen seiner Aufteilung in vier Hauptbücher mit jeweils vier Untergruppen auch als Tetrabiblon bezeichnet wird, orientiert sich am Vorbild des Oreibasios, ist aber insgesamt weniger detailliert und sorgfältig als dessen Kompilation. Die große Anzahl zum Teil auch magischer Rezepturen spiegelt die volksmedizinische Tradition jener Zeit wider. Als Arzt am Hofe Justinians I. zu wirken, dürfte sicher eine Herausforderung gewesen sein. Der gestaltungsehrgeizige Bauernsohn aus dem nördlichen Mazedonien war mit Verwaltungsaufgaben, aber auch mit der Organisation von Feldzügen und Militäreinsätzen gegen äußere und innere Feinde scheinbar so überhäuft, dass sein Biograph Prokop ihn den „schlaflosesten aller Kaiser“ nennt. Aber auch apokalyptische Erdbeben, Pestwellen und Hungersnöte forderten den Organisator auf dem Kaiserthron bis an die Grenze des Verkraftbaren. Zweifellos beanspruchte Justinian auch seinen Leibarzt. Auch dessen Gattin Theodora (Potter 2015) dürfte Aëtios beansprucht haben. Ihr ausschweifend-nymphomanes Leben, das selbst wenn nur ein Teil der Verunglimpfungen des Chronisten Prokop zutrifft manchen Flügel des kaiserlichen Hofs bisweilen in bordellartige Zustände versetzte, kann nicht ohne körperliche Folgen geblieben sein. Dass hier Ge- 
schlechtskrankheiten - die die Antike als sexuell „übertragbare“ Krankheiten nicht kannte - gehäuft vorgekommen sein dürften, liegt auf der Hand. Von „fressenden Genitalgeschwüren“ wird in den Schriften oft berichtet, ebenso von „Gewächsen“, Warzen vermutlich.

Wie Aëtios muss auch Alexandros von Tralleis (ca. 525-600), gebürtig aus Tralleis, einer Polis im kleinasiatischen Lydien und Bruder des Mathematikers und Architekten Anthemios, des Baumeisters der Hagia Sophia in Konstantinopel, der Gruppe der byzantinischen Kompilatoren zugerechnet werden. Seine ärztlich-literarische Tätigkeit ist doch um einiges selbstständiger, als die seiner bedeutenden Vorgänger. Das Hauptwerk dieses Arztes, der lange Zeit auch in Rom lebte, besteht in einer 12 Bücher umfassenden Therapeutik, die neben Rückgriffen auf die hippokratisch-galenische Medizin auch von eigener praktischer Erfahrung zeugt. Obwohl Alexandros prinzipiell dem Grundsatz des contraria contrariis folgt, schätzt er doch wie kaum ein anderer die Naturheilkraft, die man drastischen Heilkuren durchaus vorzuziehen habe. Warme und kalte Bäder, feuchte Umschläge und Bewegung sind seine therapeutischen Mittel. Erst wenn sie versagen, darf Zuflucht beim Aderlass, beim Purgieren und bei Medikamenten genommen werden. Besonderes Gewicht hat Alexandros auf die Krankheiten des Nervensystems gelegt. Bemerkenswert sind hier seine Beobachtungen zur Epilepsie, der ja Galen von Pergamon bereits den Nimbus einer heiligen Krankheit genommen und sie den Kopfkrankheiten zugewiesen hatte. Hierzu schreibt der byzantinische Arzt allerdings noch:

Der Beweis, dass Epilepsie vom Magen ausgeht, liegt darin, dass im Magen häufig eine Unruhe und ein fressendes Nagen entsteht, und dass alsdann die Kranken das Nahen des Leidens fühlen. [...] Sobald der Kranke morgens aufgestanden ist und seinen Darm entleert hat, genieße er eine Ysopabkochung, die ihm sehr nützlich ist, denn viele sind allein durch ihren Gebrauch geheilt worden, so dass sie nicht mehr als zwei- oder dreimal in diese Krankheit fielen. Unvermischten Wein nach dem Bade zu trinken ist verboten, weil nichts leichter einen Anfall auslöst - wie überhaupt allen Epileptikern unvermischter Wein gefährlich ist (Alexandros von Tralleis, Zwölf Bücher über die Medizin (Liber I, Kap. 15), hier zit. nach Puschmann 1878, S. 534-536).

Nun ist Ysop (Eisenkraut, Honigkraut, Hyssopus officinalis) keineswegs den Heilmitteln gegen die Epilepsie zuzuordnen. Aber die hebräische Bedeutung von Ysop - (אז', ésóv) für „heiliges Kraut“ - legt nahe, dass es in der Antike und in Byzanz noch gegen eine Krankheit eingesetzt wurde, deren Ausbruch man göttlichem Einfluss zuschrieb. Richtig ist, dass sich die dem epileptischen Anfall vorausgehende „Aura“ durchaus auch in Magenbeschwerden äußern kann und dass Alkohol bei Epileptikern Anfälle auslösen kann und deshalb gemieden werden sollte. Aber auch Rezepte gegen Augen- und Ohrenleiden, gegen Krank- 
heiten des Unterleibs und des Urogenital- und Darmtraktes finden sich bei Alexander. Er beschreibt darüber hinaus Fieber, Augenkrankheiten und Eingeweidewürmer, Harnleiden und die Gicht. Interessant ist sein häufiger Rückgriff auf Zaubermittel, Amulette, Gemmen und andere magische Therapeutika, wohl auch, um damit den Wünschen seiner Patienten entgegenzukommen:

Unbedingte Pflicht eines verantwortungsbewussten Arztes ist es, jegliches therapeutische Hilfsmittel in Erwägung zu ziehen. [...] Da jedoch der momentan herrschende Zeitgeist die Anwendung von Heilmitteln, die durch eine immanente Naturkraft wirken, aus Unwissenheit tadelt, habe ich es vermieden, solche andauernd zu verordnen (Grimm-Stadelmann 2019, 2020).

Tatsächlich beschrieb Alexandros neben „herkömmlichen“ Therapiemethoden ein breites Spektrum an magischen Mitteln und Heilpraktiken. Er empfahl sie oftmals als Ergänzung, mitunter aber auch als Alternative, in diesem Falle häufig auf den Wunsch der Patienten. Alexandros ging damit nicht zuletzt auf ihre psychischen Befindlichkeiten und ihre spirituellen Bedürfnisse ein. Typisch ist auch hier die Behandlung der Epilepsie, für die er magische Kuren aus dem Bereich der Similemagie und den Einsatz von Amuletten vorschlägt, so etwa den Nagel eines Kreuzes, der um den Hals getragen werden soll. Die Begründung für den Einsatz magischer Heilmittel ist pragmatisch: „Damit“, so leitet er eine Passage über Wunderkuren gegen die Epilepsie ein,

ist der Gegenstand ziemlich erschöpft; ich habe angeführt, sowohl was ich selbst über die Epilepsie wusste, als auch, was mich eine lange Erfahrung gelehrt hat. Da jedoch Manche an den Wundermitteln und an Amuletten Freude haben, dieselben anzuwenden wünschen und damit auch wirklich zum Ziele kommen, so hielt ich es für passend, mich darüber denen gegenüber, die sich dafür interessieren, auszusprechen, damit der Arzt in der Lage sei, in jeder Weise seinen Kranken zu helfen (Puschmann 1878, S. 556).

Die nun folgenden Rezepturen muten heute seltsam an, aber sie sind doch der Similemagie entnommen, die im Sinne der Sympathie auf die gleichgerichtete Wirkung durch die Applikation eines Stoffes aus der belebten oder unbelebten Materia medica setzt:

Auch folgendes Mittel übt eine wunderbare Wirkung aus. Wenn man junge Schwalben aufschneidet, so findet man in ihrem Inneren zwei Steinchen, von denen das eine schwarz, das andere weiß ist. Legt man nun das weiße Steinchen auf, sobald ein Epileptiker darniedergestürzt ist, so kommt er wieder zu sich; das schwarze Steinchen nehme man und binde es dem Kranken auf die Haut. Man erzählt, die Schwalben gäben diese Steinchen nur ihrem ersten Jungen; man findet sie deshalb nicht leicht und nur dann, wenn man sämtliche Junge öffnet (Puschmann 1878, S. 560). 
Hier wird offensichtlich auf sturzverhindernde Wirkung der magischen Steinchen der meisterlich fliegenden Schwalben gesetzt. Unklarer bleibt die Wirkung der Amulette, bei denen häufig die Lithotherapie, das Heilen mit Steinen, zur Anwendung kommt:

\begin{abstract}
Amulette müssen gegen die Epilepsie angewendet werden, wie auch Asklepiades, der bekannte Arzneibereiter, behauptet [...]. Ebenso sagt Zalachthos, dass der Jaspis, welcher auch Rauchstein genannt wird, bei allen Uebeln, die sich auf den Kopf und den Verstand werfen, umgehängt wird; wird er gerieben und dabei nass gemacht, so zeigt sich deutlich seine wunderbare Wirkung. Ferner schreibt Ostanes: man solle Korallen, Päonien und Nachtschatten-Wurzeln bei abnehmendem Monde sammeln, in ein Stück Leinwand einpacken und umhängen. Oder man trage einen gleich dem Türkis blaugrün schimmernden Jaspis am Finger, und man wird von der Krankheit geheilt werden. Derselbe hat großen Werth (Puschmann 1878, S. 566).
\end{abstract}

Frühe Übersetzungen ins Lateinische, aber auch ins Arabische, Hebräische und Syrische förderten die breite Rezeption von Alexandros' Schriften durch das gesamte Mittelalter.

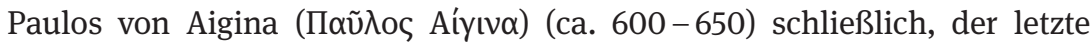
Vertreter der bedeutenden ärztlichen Schriftsteller in dieser ersten Phase der byzantinischen Medizin, war in der ersten Hälfte des 7. Jahrhunderts in Alexandria tätig. Von ihm ist uns ein siebenbändiges Handbuch der praktischen Medizin (Pragmateia) überliefert, das sich über weite Strecken an Oreibasios ausrichtet. Dieses Werk war als kompendienartiges Handbuch, vielleicht als Repetitorium gedacht und sollte insbesondere reisenden Ärzten dienlich sein. Paulos galt aber später bei den Arabern auch als Spezialist für Geburtshilfe und damit für Hebammenfragen (der „Hebammenspezialist“). Mit eigenen Auffassungen und Ergänzungen will sich der Verfasser ausdrücklich nicht einbringen. Zu seinen Themen gehören auch Anleitungen zum Verhalten in besonderen Lebenssituationen, wie etwa beim Verliebtsein und beim Sex. Die erste Liebe war für Paulos durchaus den Gehirn- und Seelenleiden zuzuordnen, gekennzeichnet von ständiger Sorge - „die Sorge ist aber ein Seelenleiden“ (Paulos von Aegina 1914, S. 192) - gleichzeitig aber auch durch ständige Überfüllung mit Vergnügen. Einige werden in der Sorge schlaflos und magern ab. Die Klügeren aber neigen zu reichlichen Bädern, zum Weingenuss, zur Gymnastik und zum Besuch von Schauspielen. Der Liebesgenuss selbst ist für Paulos durchaus anstrengende Arbeit. Deshalb ist „reichliche Ernährung“ von Nutzen (Paulos von Aegina 1914, S. 30). Feuchte Nahrung ist besonders anzuraten, also Fische, Muscheln und Meerpolypen (vermutlich Oktopusse). Auch nahrhafte Hülsenfrüchte und andere Gemüse sind zu empfehlen: Kichererbsen, Bohnen, Gartenerbsen, die Rauke, kleine Rüben. „Gar sehr empfehle ich die Trauben, denn sie machen feucht, füllen 
das Blut mit Lebenspneuma, was zum Liebesgenuß reizt“ (Paulos von Aegina 1914, S. 30). Wer den Beischlaf ausübt, muss aber von „Übersättigung und Unverdaulichkeit, von Mattigkeit, Erbrechen oder Durchfall frei“ sein (Paulos von Aegina 1914, S. 30). Auch ungezügelte Sinnenlust billigt Paulos nicht. Alles soll im rechten Maße stattfinden. - Aber auch für die altersschwachen Greise hat Paulos durchaus Ratschläge: „Das Greisenalter ist trocken und kalt; seine Auffrischung geschieht durch Mittel, die erwärmen und feucht machen“ (Paulos von Aegina 1914, S. 23). Den Schwächeren ist

dreimal (täglich) Nahrung zu geben, und zwar um die dritte Stunde [gegen 9 Uhr morgens] etwas Brot mit attischem Honig, darauf um die siebte Stunde, nachdem sie frottiert sind und die für Greise angebrachten gymnastischen Übungen gemacht haben und gebadet sind, ist ihnen solches zu reichen, was die Leibesöffnung befördert, darnach Fische und Geflügel, und ferner bei der Hauptmahlzeit [am Abend] Nahrhaftes [saftreiches] und nicht leicht Verderbendes (Paulos von Aegina 1914, S. 24).

Von besonderer Bedeutung in diesem Kompendium ist vor allem das sechste Buch, das sich ausführlich der Chirurgie widmet. Auch scheint dieses Buch in einigen Passagen durchaus selbstständig vor dem Hintergrund eigener Erfahrungen verfasst zu sein. In ihm schildert Paulos ausführlich das Ausschneiden von bösartigen und fauligen Geschwüren, Granulationen und karzinomatösen Neubildungen. Auch der Intimbereich wird nicht ausgelassen. Hämorrhoiden bindet Paulos mit dem Faden ab und verödet sie so. Eine aber soll geöffnet bleiben, damit aus ihr das schlechte Blut abfließen kann. Danach werden Ölkompressen eingelegt. Interessant ist auch der Abschnitt über die Kriegschirurgie und die ausführliche Beschreibung chirurgischer Instrumente. Im Grunde glich die Chirurgie des Paulos der modernen schon sehr. Selbst „Umschlingungen“ zur Blutstillung waren wohl bekannt, gerieten später aber wieder in Vergessenheit. Paulos schreibt dazu: Wenn es ein großer Blutfluss ist,

versuche, das Gefäß mit einem Haken in die Höhe zu ziehen und winde es mässig herum. Wenn das Blut zurückgedrängt ist, und das Gefäß eine Ader [Vene] ist, muss man versuchen, das Blut durch eben dieselben Mittel zu stillen. Wenn es aber eine Arterie ist, muss man eins von zweien tun, entweder es wird eine Schlinge angelegt, oder du wirst das ganze Gefäß durchschneiden [und abbinden] (Paulos von Aegina 1914, S. 406).

Allerdings sollte die Chirurgie bis ins 19. Jahrhundert schmutzig, schmerzhaft und blutig bleiben. Auch die inneren Körperhöhlen blieben wegen der tödlichen Sepsisgefahr ein noli me tangere.

Mit Paulos von Aigina endet die alexandrinische Phase der byzantinischen Medizin. Noch in seine Lebenszeit dürfte die Eroberung Alexandrias durch die Araber im Jahre 642 gefallen sein. Die Übernahme der Stadt bedeutete gleichzeitig 
das Ende des griechischsprachigen Unterrichts an der dortigen Medizinschule und signalisierte damit einen wichtigen Bruch in der griechischen Rezeptionstradition der antiken Medizin. Dieser Bruch erstreckte sich freilich mehr auf die sprachliche Form als auf den inneren Gehalt jener Rezeption. Die Tatsache nämlich, dass eine bekannte und wichtige ärztliche Persönlichkeit wie die des Paulos von Aigina unmittelbar an der Nahtstelle zwischen griechischer und arabischer Kultur in Alexandria gewirkt hatte, führte dazu, dass die frühe arabische Medizin an die Schriften dieses ins Arabische übersetzten Autors anknüpfte. So war der Fall des griechischen Alexandria gleichzeitig eine wichtige Voraussetzung für den späteren Rezeptionsweg der antiken Medizin über den arabischen Kulturraum zurück nach Europa.

\section{Das christliche byzantinische Hospital}

Die Idee des barmherzigen Krankenhauses in Fürsorge für „Brüder und Schwestern in Not“ findet sich erst in der christlichen Spätantike (Mt. 25, 35-40; Lk. 10, 30 -37). Asklepiosheiligtümer (seit dem 6. Jahrhundert v.Chr.) (Steger 2004), römisch-antike Krankenstuben (Iatreia bzw. tabernae medicae) oder römische Valetudinarien für das Militär folgten diesem allgemein caritativen Impuls noch nicht. Das Konzil von Nicäa (325 n. Chr.) soll zwar bestimmt haben, dass alle Bischöfe in ihrer Diözese ein Hospital einzurichten hätten. Tatsächlich aber ist dieser Beschluss auf dem Konzil selbst so nie ergangen, sondern erst im 6. Jahrhundert den Konzilsakten beigefügt worden. Sehr wohl lassen sich mischfunktionale Xenodochien, also Fremdenherbergen bis weit ins westliche Mittelalter nachweisen. Dass in diesen mittelalterlichen Hospitälern neben Armen, Fremden oder Waisen wohl auch Kranke Aufnahme fanden, ist belegt, heißt aber noch nicht, dass wir diese Institutionen als Krankenhäuser im strengen Sinne auffassen dürfen. Dies gilt für den Westen ausnahmslos bis zur Entwicklung des bürgerlichstädtischen Hospitals im 16. Jahrhundert.

Anders im griechisch-byzantinischen Osten. Dort entstehen im Zusammenhang mit einer fulminanten Christianisierungswelle seit dem 4. Jahrhundert differenzierte Einrichtungen zur Versorgung von fremden Bedürftigen (Xenodoche-

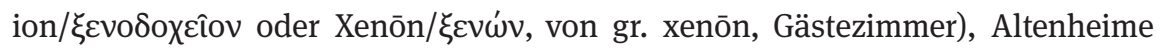
(Gēratropheion, von gr. Géron, „Greis“, und gr. trophaion, „Zufluchtsort“), Armenhäuser (Ptōcheion), Speiseausgaben für Bettler (Ptōchtropaion) und Krankenhäuser (Nosokomeia, von gr. nosos, „Krankheit“, gr. kiomaō, „sich zur Ruhe legen“) (Bennett 2017; Kidonopoulos 1994; Kotzabassi 2013). Fundament der christlichen spätantiken Polis ist die Caritas, worauf in Antiochia besonders Bischof Joannis Chrysostomos (344/49-407) in seinen Predigten immer wieder 
besteht. In den Urkunden kann aber auch durchaus der allgemeinere Begriff „Xenon“ in der Bedeutung von Krankenhaus auftauchen. Auftraggeber solcher Einrichtungen waren neben Bischöfen meist die Kaiser, die für solche Belege ihrer Philanthropie staatliche Gebäude umwidmeten oder die Funktion sakraler Institutionen, meist Klöster, um eine medizinisch-caritative erweiterten. So lässt etwa Kaiser Isaakios II. Angelos (1155-1204) um 1190 Gebäude seines ermordeten Vorgängers Andronikos I. Komnēnos (1122-1185) bei der Kirche der Vierzig Märtyrer in ein Krankenhaus (nosokomeion) umwandeln, das dann seinen Namen trägt. Die meisten solcher Stiftungskrankenhäuser waren öffentliche Krankenanstalten (Dèmosia Iatreia, Koina Iatreia), die bereits im Namen darauf hinweisen, dass Ärzte in ihnen tätig waren; so auch das in unmittelbarer Nähe des IōannēsProdromos-Klosters gelegene Krale-Krankenhaus (Xenōn tu Kralē), das von dem serbischen Schwiegersohn Kaiser Andronikos’ II. Palaiologos (1159-1232), König Stephan Uroš II. Milutin (1253-1321), für „unzählbares Geld“ gestiftet worden und auch der Medizinerausbildung gewidmet war. Das Krankenhaus verfügte über eine umfangreiche Bibliothek, die auch die berühmte (später Wiener) Handschrift des Dioskorides beherbergte. Auf dem Hof des Xenon befanden sich Wohnhäuser und eine Kirche. Diese Einrichtung bestand noch bis zur Eroberung der Stadt durch die Osmanen 1453. Als idealtypische Institution muss hier das Pantokratorkloster in Konstantinopel, heute die Molla Zeyrek Moschee im Istanbuler Stadtbezirk Fatih, erwähnt werden. Bereits im 9. Jahrhundert hatte Theophilos (829-842) dort ein aristokratisches Haus in ein Hospital mit 50 Betten umwandeln lassen, das eine Männer- und eine Frauenabteilung aufwies und über ein umfangreiches medizinisches Personal sowie über eine Bibliothek mit Handschriften antiker medizinischer Autoren und Rezeptsammlungen verfügte. Auch ein Bad gehörte dazu, zwei kleine Kirchen für Männer und Frauen getrennt, eine große Parkanlage, in der „immer ein frischer Luftzug“ wehte, sowie ein Gēratropheion. Zwischen 1118 und 1124 hatte Kaiserin Eirene Komnena (1068 - ca. 1134) dort zunächst ein Kloster erbauen lassen, zu dem eine Kreuzkuppelkirche, ein Krankenhaus und eine Bibliothek gehörten. Nach ihrem Tod ließ Kaiser Johannes II. Komnenos (1087-1143) den Gebäudekomplex noch erweitern. Ein arbeitsteiliger Ärztestab versorgte die Kranken medizinisch, also nicht nur pflegerisch. Über die tägliche Versorgungspraxis wissen wir allerdings nur das, was im jeweiligen Statut der Häuser (Xenōn typicon) dispositiv festgelegt wurde. Allerdings lassen die Texte der Statuten auf lange Organisationstraditionen schließen, die sich praktisch bewährt haben müssen.

Eine lebendige Schilderung, wie sich eine medizinische Behandlung in einem byzantinischen Xenon abspielen konnte, im vorliegenden Fall die schmerzhafte Kauterisierung (Ausbrennen) eines Geschwürs, verdanken wir dem byzantinischen Dichter Theodoros Prodromos (ca. 1100 - ca. 1157). Prodromos beschreibt 
auch die Assistenten des Arztes im Operationsraum. Einige fachten die Glut im Ofen an, andere gaben Brenneisen in die Glut, wieder andere bereiteten das Bett für Prodromos vor und die Fesseln, mit denen der arme Patient während des schmerzhaften Ausbrennens fixiert wurde. Prodromos, der die Prozedur unter der Schirmherrschaft des Hippokrates und des Galen offensichtlich überlebte, erinnert sich später:

Und über all diesen Assistenten stand der liebende Hospitaldirektor (nosokomos), der Beste
seiner gleichen, hervorragend ausgebildet, mein lieber Freund der Krankheiten-Heiler, und
noch dazu ganz ohne Bezahlung. Er scherzt mit mir, lächelt mich an, nennt mich seinen
Freund. Dies ist seine Medizin gegen die zu erwartenden Schmerzen, Witzchen machen,
Geschichten erzählen, freundlich lächeln. Ganz nebenbei beobachtet er mich, schätzt mich
gleichsam ab, dieser medizinische Herrscher, und markiert mit seinem schwarzen Stift auf
meinem Körper, wo er mich brennen wird. Und dann: Welch Schrei des Unglücks und des
Schmerzes! Dem Feuer zum Verzehr hingegeben, verbrannt zu Asche! Durch und durch im
Feuer gebacken werde ich Gott zum Opfer gebracht. Und dies keineswegs als unschuldiges
Lamm, wie ich es ja wirklich bin, sondern als verfluchter Widder (Codellas 1946, S.212).

Die Gesamtzahl der Hospitäler in Konstantinopel vom 6. bis zum 13. Jahrhundert lag nach verschiedenen Schätzungen zwischen 16 und 28, lässt sich allerdings aufgrund terminologischer Probleme nicht genau bestimmen. Zweifellos lag ihre Blütezeit aber im 11. und 12. Jahrhundert, als dort von Ärzten vielfältige stationäre Behandlungen aber auch ambulante Versorgungsdienste angeboten werden konnten. Auch die ärztliche Ausbildung fand in einem besonderen Seminar (gr. phrontistērion, Denkort) bestimmter Hospitäler Konstantinopels statt, so etwa am Pantokrator-Spital, wo eine Art Medizinischer Direktor (gr. tēs iatrikēs proexarchōn, Ärztevorsteher) die Prüfungen der jungen Ärzte abnahm und ihnen bei Bestehen eine Auszeichnung (gr. symbolon) verlieh. Gleiches galt sicher nicht nur für Konstantinopel, sondern für jede größere Stadt in der Provinz, so etwa im Kontext der Xenodochien und Nosokomien von Antiochia, Apameia, Edessa oder Emesa, im Bereich der ostsyrischen Kirche oder auf Zypern. - Insgesamt steht das byzantinische Hospitalwesen in erster Linie für einen hohen Grad an praktizierter Philanthropie mit ihrer Sorge um Menschen in körperlicher und sozialer Bedürftigkeit. Für ein generelles Urteil über den Grad der Medikalisierung der byzantinischen Gesellschaft reicht die begrenzte Quellenlage allerdings nicht aus, auch wenn Kaiser Nikephoros Phokas (912-969) im 10. Jahrhundert ein Gesetz erließ, das den weiteren Bau von Krankenhäusern verbot, weil das Reich bereits über hinreichend viele solcher Institutionen verfüge. Wir wissen von etwa 150 bis 170 insgesamt. 


\section{Die zweite Phase der byzantinischen Medizin}

Nach dem Fall Alexandrias wird Konstantinopel zum Zentrum der zweiten Phase der byzantinischen Medizin. Entscheidend für diese Phase ist ihre starke Orientierung an praktischen Interessen der klinischen Medizin. So ist in den meisten der erhaltenen Schriften die ärztliche Diagnostik durch eine starke Gewichtung der Uroskopie und der Pulslehre betont, aber auch umfangreiche pharmazeutische Sammlungen, Abhandlungen über Nahrungsmittel, Kräuter und Gewürze bestimmen das Bild. Typisch für die zweite Phase der byzantinischen Medizin ist auch das enge Ineinandergehen von Erfahrungselementen der eigenen Kulturtradition und solchen aus der arabischen Welt, die ihrerseits auf alte vorderasiatische Überlieferungstraditionen zurückgreifen.

Die Ärzte Psellos und Niketas gehören in der ersten Hälfte dieser Phase als Autoren des 11. Jahrhunderts sicher zu den wichtigsten schreibenden Vertretern ihrer Zunft, vielleicht auch noch Simeon Seth, der allerdings unter dem Verdacht steht, als Quellen in erster Linie die Schriften seines Zeitgenossen Psellos unter seinem Namen veröffentlicht zu haben. Aber das war durchaus nicht unüblich. Psellos scheint in jener ersten Gruppe immerhin die markanteste Persönlichkeit gewesen $\mathrm{zu}$ sein. $\mathrm{Zu}$ den wichtigsten Werken - die das größte medizinische Schriften-Corpus der byzantinischen Literatur überhaupt bilden (Leven 1987) des Konstantinos (Michael) Psellos (1017/18 - ca. 1078) - Jurist, Politiker und Universalgelehrter an der philosophisch orientierten Hochschule in Konstantinopel, den wir auch als Lehrer und Erzieher des byzantinischen Kaisers Michael VII. Dukas (1050 - ca. 1090) identifizieren können -, gehören neben einer allgemeinen Enzyklopädie eine Synopsis der gesamten Heilkunst, Fragmente eines Kompendiums der gesamten Medizin, Abhandlungen über Zeugungs- und Vererbungsvorstellungen, über Epilepsie, Schwachsinn und Klugheit sowie ein Traktat über die Heilkräfte von Edelsteinen und über Dämonen. Bedeutsam ist seine Arzneimittellehre, von der allerdings noch nicht letztgültig geklärt ist, ob sie hauptsachlich Psellos oder dem Simeon Seth („Symeōn, ein Meister aus Antiochia, Sohn des Sēth“, um 1050, einem jüdischen Arzt-Gelehrten, Astrologen und Protovestiarius (Oberschatzmeister) unter Kaiser Michael VII. Dukas) zugeschrieben werden kann. In dieser bedeutsamen Materia medica sind abendländische Rezeptoren ebenso wie solche aus dem arabischen Kulturkreis enthalten. Von Niketas schließlich, der als Arzt um 1100 tätig war, besitzen wir eine Kompilation chirurgischer Texte aus der klassischen und byzantinischen Zeit der griechischen Medizin, in die neben chirurgischen Texten des Hippokrates, des Galen und vieler weniger bekannter Ärzte auch eigene Erfahrungen eingegangen sein dürften. 
Die Ärzte des 13. und 14. Jahrhunderts, Nikolaos Myrepsos („Der Salbenkoch“, spätes 13. Jh.) und Johannes Aktuarios (ca. 1275-1328), gehören zu den bedeutenden Vertretern der Endphase der byzantinischen Medizin. So besitzen wir von Myrepsos eine mehr als 2600 Rezepte umfassende Sammlung (Antidotarium) von Arzneivorschriften, einen ungeheuren Schatz ärztlich-pharmazeutischen Wissens, der freilich enge Anknüpfungspunkte an eine ähnlichen Sammlung aus der lateinischen Tradition Salernos aufweist. Seine Sammlung diente in Frankreich noch bis ins 17. Jahrhundert hinein als offizielle Pharmakopöe. Von Johannes Aktuarios ist uns eine Therapeutik überliefert, die neben Rückgriffen auf alte Texte reich an eigenen praktischen Erfahrungen ihres Verfassers ist. Aktuarios scheint aber auch ein ausgezeichneter Kenner der klassischen Philosophie und insbesondere der Seelentheorie des Aristoteles gewesen zu sein. Interessant ist eine Abhandlung von den Seelenfunktionen, die die antiken Vorstellungen zusammenfasst, ausdifferenziert und darüber hinaus Beziehungen zwischen Geistestätigkeit und diätetischer Lebensführung herstellt.

\section{Exkurs: Gottes Strafe - Die Justinianische Pest}

Inmitten der größten Bedrohung des Reichs durch den Einfall der Sassaniden unter Chosrau I. von Persien, der im März 540 Antiochia eroberte, rücksichtslos plünderte und nur durch hohe Gegenleistungen in Gold zum Rückzug bewegt werden konnte, traf Byzanz eine mächtige Epidemie (Leven 1987; Little 2009; Meier 2016, 2020). Durch neuere archäogenetische Studien inzwischen unzweifelhaft belegt, handelte es sich bei ihr um die durch Yersinia pestis ausgelöste Pest, sehr ähnlich dem Schwarzen Tod des 14. Jahrhunderts. An tödlicher Virulenz ließ diese Seuche der späten Regierungszeit des Justinian nichts zu fürchten übrig. In den Straßen türmten sich die Leichen. Hauptquelle für die Dramatik, mit der die Krankheit das Reich in Afrika, Südosteuropa und Kleinasien heimsuchte, sind Augenzeugenberichte des Historikers Prokop von Caesarea (500 562) und Anmerkungen ex post des Kirchenhistorikers Eugarios Scholasticos (536/ 37- ca. 600) sowie des Historikers Agathias von Myrina (531/32- ca. 582). Ärztliche Zeitzeugen der Justinianischen Pest waren die bereits genannten Paulos von Aegina und Alexandros von Tralleis. Über die große Pest, die sie als Jugendliche erlebt haben müssen, berichten sie jedoch nicht. Vielleicht war es aber die Seuche, die sie zum Studium der Krankheiten motiviert hatte.

Ausgebrochen 541 in Oberägypten verbreitete sich die Pest durch den Schiffsverkehr schnell über das ganze Reich. Die Todesrate der Seuche, die sich scheinbar überwiegend als Beulenpest manifestierte, muss enorm gewesen sein und überschritt die Millionengrenze vermutlich um ein Vielfaches, wenngleich die 
zum Beleg angeführten Zahlenangaben im Detail (10.000 Tote pro Tag) heute deutlich überzogen wirken. Die Bedeutung extrem hoher Zahlen ist in der Spätantike nicht statistisch zu verstehen, sondern metaphorisch. Gleichwohl muss der demographische Einschnitt durch diese Seuche erheblich gewesen sein, zumal sie in zeitlichen „Wellen“ bis ins 8. Jahrhundert immer wieder auftrat.

Dass die Krankheit, vor der auch die Ärzte hilflos kapitulierten, Angst und Schrecken bis hin zur allgemeinen Panik verursachte, verwundert kaum. Vollzog sich der Tod still in den Häusern, blieben die Pestleichen oft lange unentdeckt. Am meisten demoralisierte jedoch das allen erkennbare öffentliche Sterben im Pest-Delirium. Prokop berichtet:

Diejenigen, die die Krankheit mit einem Delirium schlug, die schliefen nicht mehr, wurden wahnsinnig, fühlten sich von bösen Männern verfolgt, versuchten aufs höchste erregt zu fliehen und schrien in schrillsten Tönen. Ihre Betreuer durchlebten tiefste Erschöpfung. [...] Die nahe am Wasser lebten, wollten sich hineinstürzen; nicht etwa weil sie durstig gewesen wären, sondern weil ihnen die Krankheit den Geist vollkommen verwirrt hatte (übers. aus dem Englischen, Procopius/Dewing, XXII, 17-23, S. 459).

Die Ärzte, die die Bubonen (Achsel- oder Leistengeschwüre) der Kranken untersuchten, dürften selbst bald erkrankt sein. „Manche der Erkrankten“, so berichtet Prokop,

verstarben auf der Stelle, andere nach Tagen, manche aber wurden am ganzen Körper von linsengroßen schwarzen Pusteln übersät, solche überlebten den ersten Tag ihrer Erkrankung nicht. Wieder andere erbrachen ohne erkennbaren Anlass Blut im Schwall und fielen danach tot um (übers. aus dem Englischen, Procopius/Dewing, XXII, 30 - 36, S. 463).

Von Prokop erfahren wir, dass die Krankheit in Byzantion (Konstantinopel) vier Monate wütete, im ganzen Kaiserreich, in dem angeblich kein erreichbarer Ort verschont blieb, sicher mehrere Jahre mit vielen späteren Ausbrüchen. Die Sterblichkeit stieg allmählich an und erreichte schließlich nie gekannte Gipfel. Genauere Sterblichkeitsraten dürfen wir den scheinbar präzisen Aussagen Prokops (fünf bis 10.000 Tote täglich) schon aus demographisch-mathematischen Gründen wohl nicht entnehmen. Der Chronist unterstreicht mit solchen Zahlen nur die Unvorstellbarkeit der Geschehnisse. Sicher ist nur, dass die immense Sterblichkeit das soziale Gefüge der Stadt vollkommen auf den Kopf stellte. Individuelle Grabstätten wichen Massengräbern, Gräber wurden ohne Rücksicht auf die Angehörigen unter Einsatz von Gewalt aufgefüllt. Die Frömmigkeit ließ zu wünschen übrig: 
Das Durcheinander war total. Nicht zuletzt, weil die Sklaven häufig ihrer Herren beraubt waren, die Herren wiederum entbehrten der Dienste ihrer Sklaven, weil diese krank oder tot waren. Sogar hochangesehen Bürger blieben tagelang unbestattet. (übers. aus dem Englischen, Procopius/Dewing, XXIII, 4-10, S. 465-466)

Chaos entstand auch in Glaubensfragen. Einige wurden plötzlich sehr fromm, andere ließen alle guten Sitten fallen und ,verfielen in Schurkereien und Gesetzlosigkeiten aller Art (übers. aus dem Englischen, Procopius/Dewing, XXIII, 15-19, S. 471-472). Andererseits fanden die rivalisierenden Parteien der Stadt in der Not zusammen und halfen sich gegenseitig beim Begraben ihrer Toten.

Und wie reagierte Justinian? Er ließ Geld verteilen (auch aus seinem Privatschatz) und neue Gräber ausheben, und als dies alles nicht mehr half, ließ er die Befestigungstürme bei Galata mit Leichen auffüllen, woraufhin bald ein unerträglicher Gestank die Stadt erfüllte. Auf dem Höhepunkt der Seuche kam das gesamte Leben in Byzantion fast zu erliegen, so dass am Ende kein Handwerk mehr betrieben, auch kein Brot mehr gebacken wurde und neben der Seuche am Ende eine Hungersnot wütete, die selbst nur schwach Erkrankten dahinraffte. Auch in ihrer politischen Bedeutung darf die Pest nicht unterschätzt werden. So führte der Umstand, dass Justinian selbst wochenlang an ihr litt, zu Geheimabsprachen von Generälen im Osten des Reichs über die Nachfolge des Kaisers. Prokop selbst kolportiert auch innenpolitisch virulente Gerüchte, dass eine Seuche von Gott über das Reich gekommen sei, um den frevelhaften Kaiser und seine Gattin Theodora zu strafen (Leven 1987). Aber was war überhaupt in einem Reich noch rational nachzuvollziehen, das sich durch katastrophale Seuchen, Erdbeben wie in Antiochia im Mai 526 und die massive Bedrohung seiner Außengrenzen durch Fremdvölker - in Italien kämpften erfolgreich die Goten unter Totila, Armenien wurde durch die Sassaniden heimgesucht - permanent in Dimensionen bedroht sah, die die Menschen einfach nicht mehr verstanden.

\section{Schluss}

Insgesamt verdankt die westliche Welt der byzantinischen Medizin die Überlieferung der antiken gelehrten Heilkunst in ihrer Originalsprache, dem Griechischen. In der unmittelbaren Phase nach dem Untergang des Reichs 1453 bemühten sich westeuropäische Humanisten - exemplarisch der byzantinischgriechische Forschungsreisende Andreas Johannes Laskaris (1445-1534) im Auftrage Lorenzo de' Medicis, bereitwillig unterstützt durch den osmanischen Sultan Bayezid II. (1447/48-1512) - um die Auffindung wissenschaftlicher byzantinischer Manuskripte. So erwarb Laskaris solche Manuskripte auf For- 


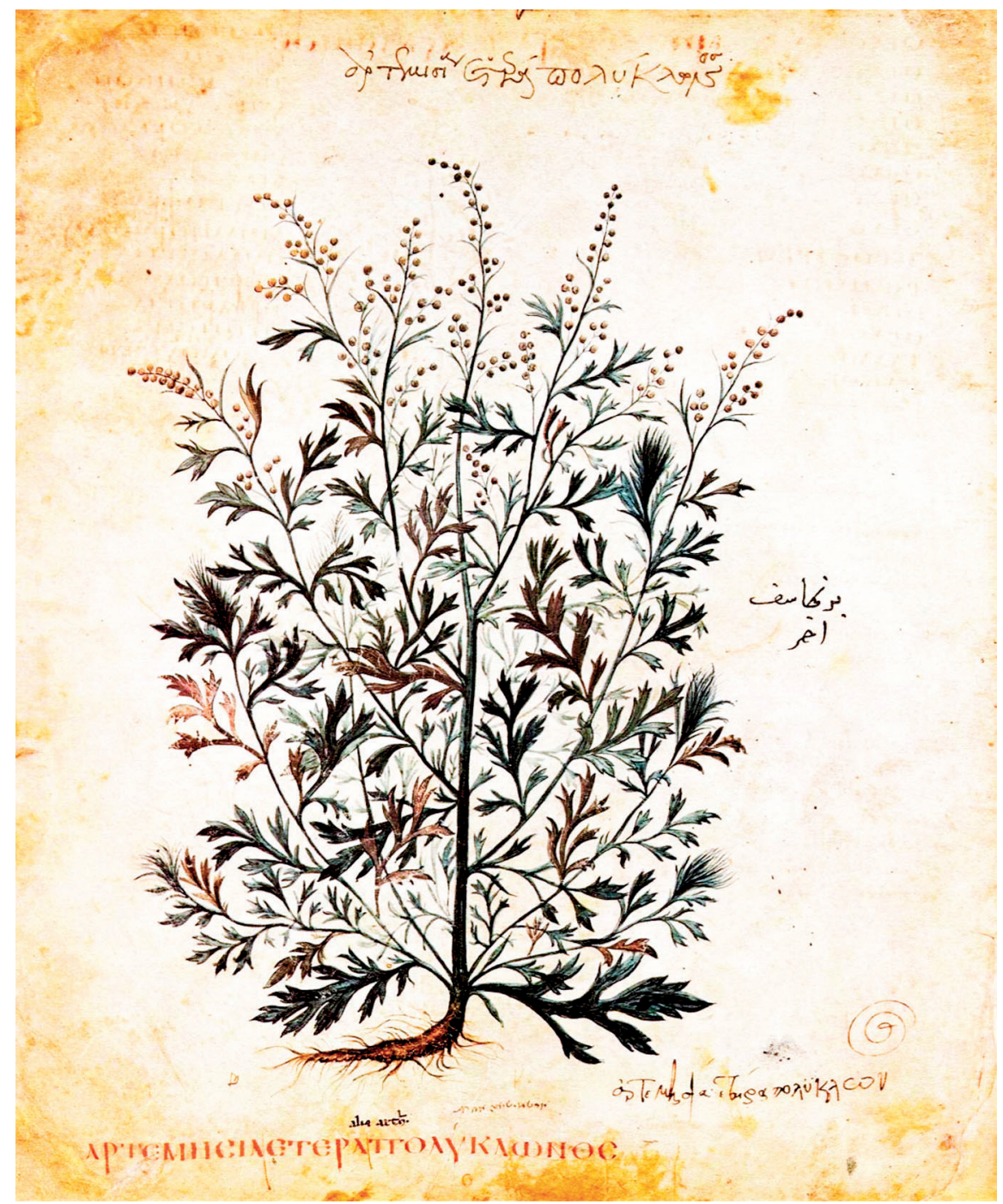

Abb. 2: Pflanzenbild (Beifuß) aus dem Wiener Dioskurides (fol. 20 verso). Der gemeine Beifuss (Artemisia vulgaris), schon im 11. Jahrhundert als „Mutter aller Kräuter“ (mater herbarum) bezeichnet, galt als Hauptmittel zur Behandlung von Frauenkrankheiten, daneben sollte er aber auch bei Verdauungsstörungen und Harnstauung helfen; besonders fette Speisen wurde mit ihm gewürzt. (Bildsammlung IGEM, Heidelberg)

schungsreisen durch den Peloponnes besonders aber in Athosklöstern und in Konstantinopel. Nun begann in Westeuropa der philologische Vergleich dieser Kodizes mit der arabisch-lateinischen Überlieferung der antiken Wissensbestände 
nicht nur der Medizin, die durch den mehrfachen Sprachtransfer zum Teil erheblichen Veränderungen unterworfen gewesen waren. $\mathrm{Zu}$ den prächtigsten noch erhaltenen byzantinischen Kodizes gehört der „Wiener Dioskurides“: insgesamt 485 Pergamentblätter mit Auszügen aus dem Arzneibuch des Pedanios Dioskurides (1. Jahrhundert) und aus heilpflanzenkundlichen Texten anderer antiker Autoren, illustriert mit farbigen Darstellungen von Pflanzen, Tieren und bedeutenden Ärzten wie Dioskurides und Galen (Abb. 3 und 4). Das Werk entstand um 512 im Auftrag einer Aristokratin, der Nobilissima Patricia Anicia Juliana (450 532). Im 12. Jahrhundert gehörte es zum Bestand der Bibliothek des Krankenhauses „Xenōn tu Kralē“ bzw. des Petra-Klosters, nach der Eroberung Konstantinopels durch Sultan Süleyman I. (1520 - 1566) gelangte es in den Besitz seines Leibarztes. Nach dessen Tod erwarb es der französische Humanist, Diplomat und Botaniker Augerius von Busbeck (Ogier Ghislain de Busbecq, 1522-1592) für 100 Golddukaten. Dieser stiftete es schließlich der Wiener Hofbibliothek.

\section{Literatur}

Arbesmann, Rudolph (1954): „The Concept of ,Christus Medicus“ in St. Augustine“. In: Traditio 10, S. $1-28$.

Bennett, David (2017): Medicine and Pharmacy in Byzantine Hospitals - A Study of the Extant Formularies. New York, NY: Routledge.

Codellas, Pan. S. (1946): „The Case of Smallpox of Theodorus Prodromus (XIIth Cent. A. D.)“. In: Bulletin of the History of Medicine 20(2), S. 207-215.

Congourdeau, Marie-Hélène (2004): „La médecine byzantine: Une réévaluation nécessaire“. In: La Revue du Praticien 54(15), S. 1733-1737.

Constantelos, Demetrios J. (1976): „A Note on ,Christos Philanthropos‘ in Byzantine Iconography“. In: Byzantion 46(1), S. 9-12.

Damian of Sinai [Erzbischof] (2007): „The Medical Saints of the Orthodox Church in Byzantine Art“. In: Michael Grünbart/Ewald Kislinger/Anna Muthesius/Dionysios Ch. Stathakopoulos (Hrsg.): Material Culture and Well-Being in Byzantium (400-1453). Proceedings of the International Conference (Cambridge, 8 - 10 September 2001). Wien: Österreichische Akademie der Wissenschaften, S. 41-49, https://www.austriaca.at/ 0xc1aa5576\%200x00178c45.pdf, besucht am 12.1.2021.

Edelstein, Emma Jeanette/Edelstein, Ludwig (1945): Asclepius: A Collection and Interpretation of the Testimonies. Bd. 2. Baltimore, MD: The Johns Hopkins Press.

Gollwitzer-Voll, Woty (2007): Christus Medicus - Heilung als Mysterium. Interpretationen eines alten Christusnamens und dessen Bedeutung in der Praktischen Theologie. Paderborn: Ferdinand Schöningh.

Grimm-Stadelmann, Isabel (2019): „Alexander von Tralleis - Arzt und Magier: Heilen mit Amulett und Astrologie“. Spektrum der Wissenschaften online. https://www.spektrum.de/ magazin/alexander-von-tralleis-arzt-und-magier/1647236, besucht am 12.11.2020. 
Grimm-Stadelmann, Isabel (2020): Untersuchungen zur latromagie in der byzantinischen Zeit. Zur Tradierung gräkoägyptischer und spätantiker iatromagischer Motive. Berlin \& Boston: De Gruyter.

Grumel, Venance (1949): „La profession médicale à Byzance à l'époque des Comnènes“. In: Revue des études byzantines 7, S. 42-46.

Helm, Jürgen (2005): „Magie“. In: Antike Medizin. Ein Lexikon. HG. von Karl-Heinz Leven, München: C. H. Beck, Sp. 581-583.

Horandner, Wolfram (1974): Theodoros Prodromos. Historische Gedichte. Wien: Akademie der Wissenschaften.

Kidonopoulos, Vassilios (1994): Bauten in Konstantinopel,1204-1328: Verfall und Zerstörung, Restaurierung, Umbau und Neubau von Profan- und Sakralbauten. Wiesbaden: Harrassowitz.

Knipp, David (1998): „Christus Medicus“ in der frühchristlichen Sarkophagskulptur. Ikonographische Studien zur Sepulkralkunst des späten vierten Jahrhunderts. Leiden: Brill.

Kotzabassi, Sofia (Hrsg.) (2013): The Pantokrator Monastery in Constantinople. Berlin \& Boston: De Gruyter.

László, Józsa (2010): „Mágikus és Vallásos Gyógyítás Bizáncban“. In: Communicationes de historia artis medicinae 210-213, S. 171-185.

Leven, Karl-Heinz (1987): „Die ,Justinianische Pest““. In: Jahrbuch des Instituts für Geschichte der Medizin der Robert-Bosch-Stiftung 6, S. 137-161.

Little, Lester K. (Hrsg.) (2009): Plague and the End of Antiquity. The Pandemic of 541-750. Cambridge: Cambridge University Press.

Markschies, Christoph (2005): „Heil und Heilung in der Spätantike“. In: Humboldt Spektrum 2, S. $1-4$.

Markschies, Christoph (2016): Das antike Christentum. Frömmigkeit, Lebensformen, Institutionen (3. Aufl.). München: C. H. Beck.

Meier, Mischa (2016): „The ,Justinianic Plague‘: The Economic Consequences of the Pandemic in the Eastern Roman Empire and Its Cultural and Religious Effects“. In: Early Medieval Europe 24(3), S. 267-292.

Meier, Mischa (2020): „The ,Justinianic Plague‘: An ,Inconsequential Pandemic‘? A Reply“. In: Medizinhistorisches Journal 55(2), S. 172.

Miller, Timothy S. (1997): The Birth of the Hospital in the Byzantine Empire (2. Aufl.). Baltimore, MD: The Johns Hopkins University Press.

Paulos von Aegina (1914): Des besten Arztes sieben Bücher. Leiden: Verlagsbuchhandlung vormals E. J. Brill.

Potter, David Stone (2015): Theodora. Actress, Empress, Saint. Oxford Oxford University Press.

Procopius (1914): Procopius with an English Translation by H.B. Dewing, Bd. 1, London: William Heinemann, S. 451-473 (= History of the Wars, II. xxii-xxxiii).

Puschmann, Theodor (1878): Alexander von Tralles: Original-Texte und Übersetzung nebst einer einleitenden Abhandlung. Ein Beitrag zur Geschichte der Medicin. Bd. 1. Wien: Braumüller.

Risse, Guenter B. (2011): Mending Bodies, Saving Souls - A History of Hospitals. Oxford: Oxford University Press.

Rothschuh, Karl Eduard (1978): Iatromagie Begriff, Merkmale, Motive, Systematik. 223. Sitzung am 20. Juli 1977 in Düsseldorf. Opladen: Westdeutscher Verlag. 
Schreiner, Peter (2006): „Aspekte der politischen Heiligenverehrung in Byzanz“. In: Peter Schreiner (Hrsg.): Byzantinische Kultur. Eine Aufsatzsammlung, Bd. 2. Rom: Edizioni di storia e letteratura, S. 365-383.

Steger, Florian (2004): Asklepiosmedizin - Medizinischer Alltag in der römischen Kaiserzeit. Stuttgart: Franz Steiner.

Temkin, Owsei (1962): „Byzantine Medicine: Tradition and Empiricism“. In: Dumbarton Oaks Papers 16, S. 95-115.

Vikan, Gary (1984): „Art, Medicine, and Magic in Early Byzantium“. In: Dumbarton Oaks Papers 38 , S. $65-86$. 